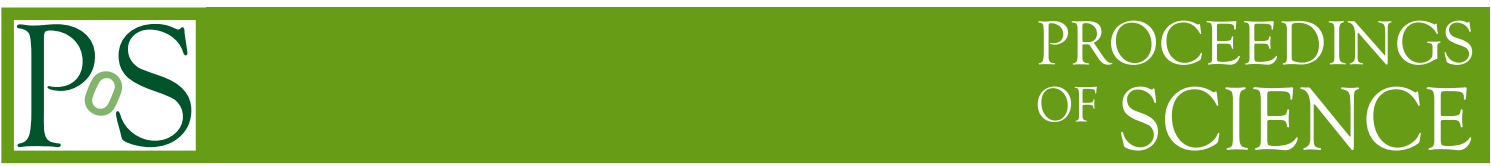

\title{
MuPix8 - A large-area HV-MAPS chip
}

H. Augustin ${ }^{* a}$, N. Berger ${ }^{b}$, S. Dittmeier ${ }^{a}$, F. Ehrler ${ }^{c}$, J. Hammerich ${ }^{a}$, A. Herkert ${ }^{a}$, L. Huth ${ }^{a}$, D. Immig ${ }^{a}$, J. Kroeger ${ }^{a}$, I. Perićc ${ }^{c}$ M. Prathapan ${ }^{c}$, R. Schimassek ${ }^{c}$, A. Schöning ${ }^{a}$, A. L. Weber ${ }^{a c}$, D. Wiedner ${ }^{a}$, H. Zhang ${ }^{c}$

${ }^{a}$ Physikalisches Institut, Universität Heidelberg, Im Neuenheimer Feld 226, 69120 Heidelberg, Germany

${ }^{b}$ Institut für Kernphysik, Johannes Gutenberg-Universität Mainz, Johann-Joachim-Becherweg 45, 55128 Mainz, Germany

${ }^{c}$ Institut für Prozessdatenverarbeitung und Elektronik, KIT, Hermann-von-Helmholtz-Platz 1, 76344 Eggenstein-Leopoldshafen, Germany E-mail: heiko.christian.augustin@cern.ch

The architecture of the first large area $2 \times 1 \mathrm{~cm}^{2}$ MuPix 8 prototype, produced in an AMS $180 \mathrm{~nm}$ HV-CMOS process, is presented. The MuPix8 chip is a High Voltage Monolithic Active Pixel Sensor (HV-MAPS) being developed for the Mu3e experiment which will search for the lepton flavour violating decay $\mu^{+} \rightarrow e^{+} e^{-} e^{+}$with an unprecedented sensitivity of one in $10^{16}$ decays. To reach this sensitivity goal an ultralight-pixel tracker with $10^{-3}$ radiation lenghts per tracking layer and high rate capability is being built. The Mu3e pixel tracker will be based on MuPix chips with a thickness of $50 \mu \mathrm{m}$ and a pixel size of $80 \times 80 \mathrm{um}^{2}$. The hits are readout by on-chip state machines and the data are streamed out via four $1.25 \mathrm{Gbit} / \mathrm{s}$ data links. The MuPix8 is the first prototype which fulfills all above requirements and features the full column length of the final chip. In addition, it implements circuitry providing pulse height information, thus allowing for timewalk suppression aiming at a time resolution of $10 \mathrm{~ns}$ or better.

The 26th International Workshop on Vertex Detectors

10-15 September, 2017

Las Caldas, Asturias, Spain

${ }^{*}$ Speaker.

๑) Copyright owned by the author(s) under the terms of the Creative Commons 


\section{Introduction}

To achieve the goals of the Mu3e experiment [1] an ultra-thin pixel tracker with $1 \%$ of radiation length per layer is being build which detects up to $10^{9}$ muon decays per second. Currently used technologies can not suffice both the material and high rate conditions at the same time.

The technology enabling to build this type of detector was found in the High Voltage Monolithic Active Pixel Sensors (HV-MAPS) [2](Fig.1(a)), which are produced in a commercially available HV-CMOS process. The combination of drift based charge collection and integrated readout on the same chip allows to build fast pixel detectors with an excellent fill-factor. Further the thin active depletion region allows a thinning of the sensors to $50 \mu \mathrm{m}$ thickness.

A series of small prototypes, the so-called MuPix chips, have been designed and tested to show the feasibility of the technology. The development culminated in the MuPix7 chip which is the first fully monolithic chip and showed an excellent performance. [3-7] For the experiment a sensor with an active area of $2 \times 2 \mathrm{~cm}^{2}$ is required. In the following the architecture and novelties of the first large scale HV-MAPS prototype MuPix8 are presented. Furthermore the module prototyping which is possible with MuPix8 is highlighted.

\section{The MuPix8 Chip}

\subsection{The Architecture}

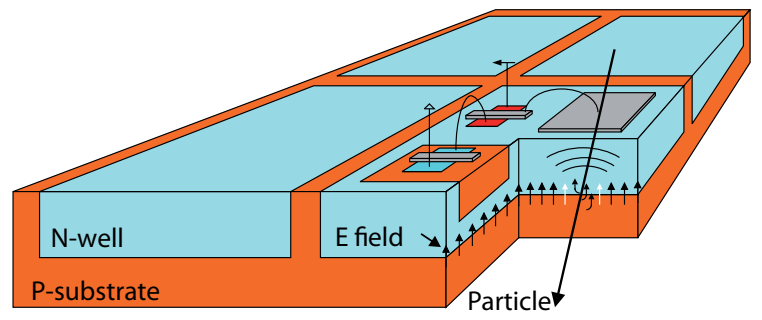

(a) Sketch of the HV-MAPS principle. [2]

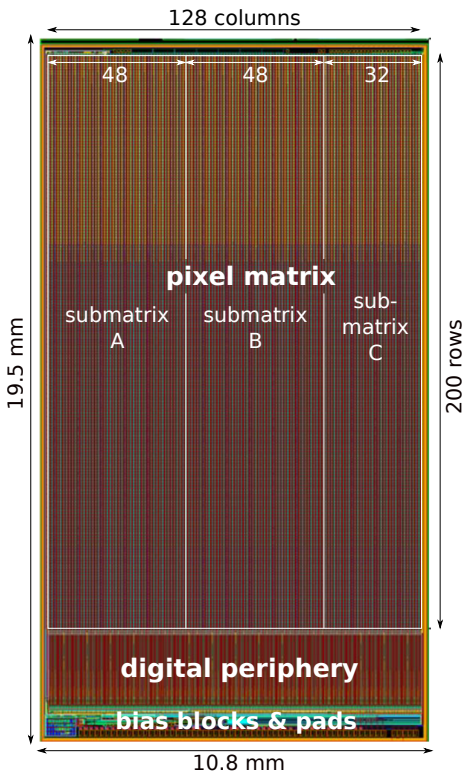

(b) The MuPix8 chip. [8]

Figure 1: The MuPix8 - A large-area HV-MAPS chip. 
The MuPix8 chip is produced in an AMS aH18 engineering run with a minimal gate length of $180 \mathrm{~nm}$ together with further test chips. Table 1 compares the design parameters of MuPix8 and MuPix7. The most notable change is the size of the prototype which enables the investigation of the column-drain architecture with the full column length of the final chip. Further the large pixel matrix of MuPix 8 is divided into three sub-matrices which are read out individually by three statemachines, which have been synthesized in one block.

The new prototype makes use of an $80 \Omega \mathrm{cm}$ substrate, four times larger than for the previous prototypes, which will increase the depletion zone approximately by a factor 2 and thereby increase the number of signal electrons for traversing charged particles. A further important change to previous prototypes is the use of enclosed transistors for analogue and biasing circuits which is expected to improve the stability against total ionizing dose effects caused by irradiation.

The overall structure is very similar to MuPix7. It can be split into three functional parts as depicted in Fig.2. Inside the active pixel matrix a charge sensitive amplifier and a signal line driver are implemented in the pixel diode. Each individual pixel has a pointto-point connection to its corresponding digital cell in the periphery. Here the signal is discriminated and a hit flag is stored along with a 10 bit timestamp. An additional 6 bit timestamp is implemented for the first time, which allows to measure the length of the analog pulse (see sec. 2.3). The information is stored until it is read out by the statemachine in a prority encoded scheme. The data is then 8bit/10bit encoded and serialized for transmission on the LVDS outputs, which are designed for transmission speed of maximally $1.6 \mathrm{Gbit} / \mathrm{s}$.

\begin{tabular}{lcc}
\hline Chip & MuPix7 & MuPix8 \\
\hline Substrate resistivity & $20 \Omega \mathrm{cm}$ & $80 \Omega \mathrm{cm}$ \\
Chip size & $0.35 \times 0.4 \mathrm{~cm}^{2}$ & $1 \times 2 \mathrm{~cm}^{2}$ \\
Matrix size & $40 \times 32$ pixels & $128 \times 200$ pixels \\
Pixel size & $103 \times 80 \mu \mathrm{m}^{2}$ & $81 \times 80 \mu \mathrm{m}^{2}$ \\
\hline
\end{tabular}

Table 1: A comparison of MuPix7 and MuPix8.

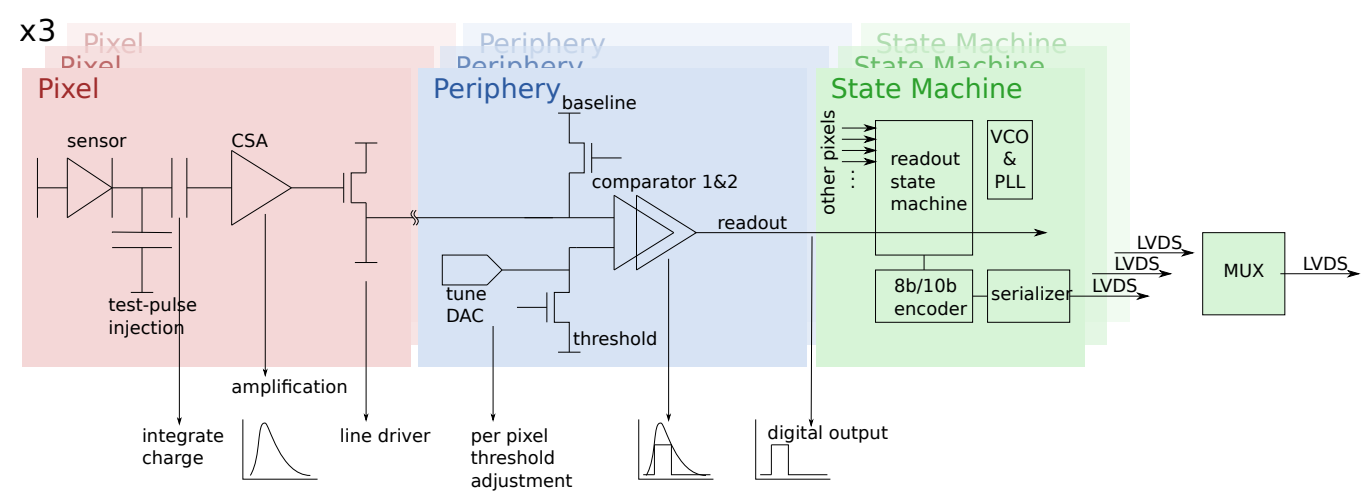

Figure 2: A functional description of the MuPix architecture. 


\subsection{Analogue Pulse Transmission \& Crosstalk}

For the MuPix7 crosstalk was observed between the transmission lines from the inpixel electronics to the periphery. The crosstalk is caused by the spatial proximity of these metal lines in the chips routing scheme [4]. For MuPix8, which is now exploring the full column length of $2 \mathrm{~cm}$ and an even higher routing density of the signal lines, this effect is expected to be even more prominent with a source follower as driver, as implemented in MuPix7. To counteract this effect a new current based transmission scheme, sketched in Fig.3, is implemented which is expected to reduce the overall crosstalk as the voltage amplitude of signal will be reduced [9]. This new approach is implemented in sub-matrix $\mathrm{B}$ and $\mathrm{C}$ of MuPix8, while Matrix A is making use of the MuPix7-like source follower, see Fig.1(b).

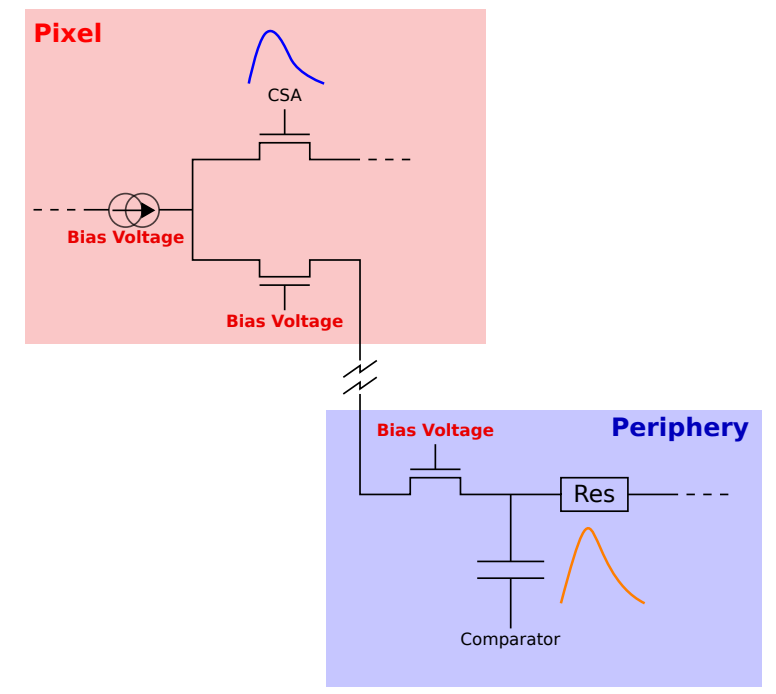

Figure 3: The current driven signal transmission.

\subsection{Timewalk Compensation}

For the MuPix7 timewalk was observed to smear out and therefore reduce the time resolution. By measuring the pulse height or an equivalent value, the hit timestamp can be corrected for timewalk. The MuPix 8 is the first prototype that samples a 6 bit pulse height information for each pixel hit and allows for off-chip timestamp correction.

The digital pixel cell contains two comparators with an individual threshold which can be used in three different modes. The simplest mode measures a Time-over-Threshold (ToT) for the discriminated pulse with a constant threshold. A more involved pulse height measurement is depicted in Fig.4(a) which makes use of a voltage ramp. The nearly perpendicular crossing angle between the ramp and signal pulse will enable a ToT measurement with less jitter, as it is less effected by noise.

The third method presented in Fig.4(b) is not relying on the pulse height measurement, but is reducing timewalk effects directly on-chip. By choosing one threshold close to the baseline to sample the timestamp, the effect of varying rising edges is minimised. The second threshold is then used to discriminate against noise. 


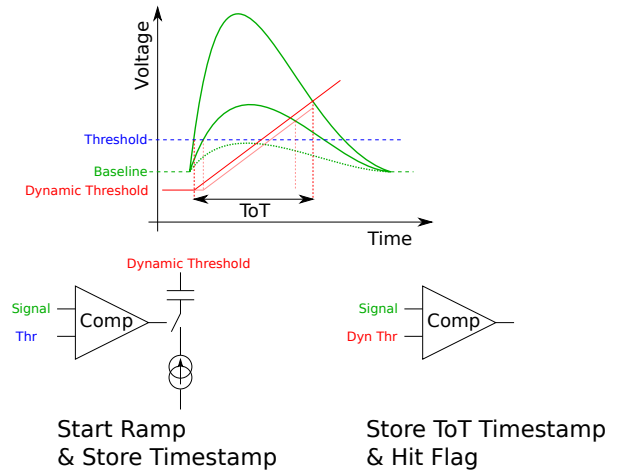

(a) ToT measurement with a voltage ramp.

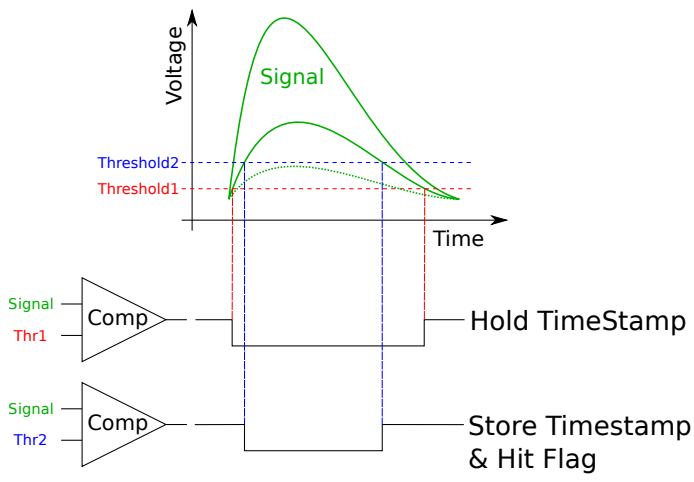

(b) The two-threshold-approach.

Figure 4: Digital pixel cell: sampling modes.

The MuPix8 is used to investigate the performance of the different modes and to decide which is most suitable for the final sensor.

\subsection{First Commissioning}

The prototype is currently commissioned and under investigation. First tests with radioactive sources showed that the chip can be configured, is responsive and can be read out. A first hitmap produced with a Strontium-90 source and a collimator is shown in Fig.5. The result demonstrates the functionality of the sensor. The difference that can be observed between Matrix $\mathrm{A}$ and $\mathrm{B}+\mathrm{C}$ originated from the different signal transmission schemes, as they generate different pulse heights in the periphery, but a global threshold is applied to all comparators independent of the matrix type. The pulses transmitted by the source follower are smaller and therefore more likely to be rejected by the comparator.

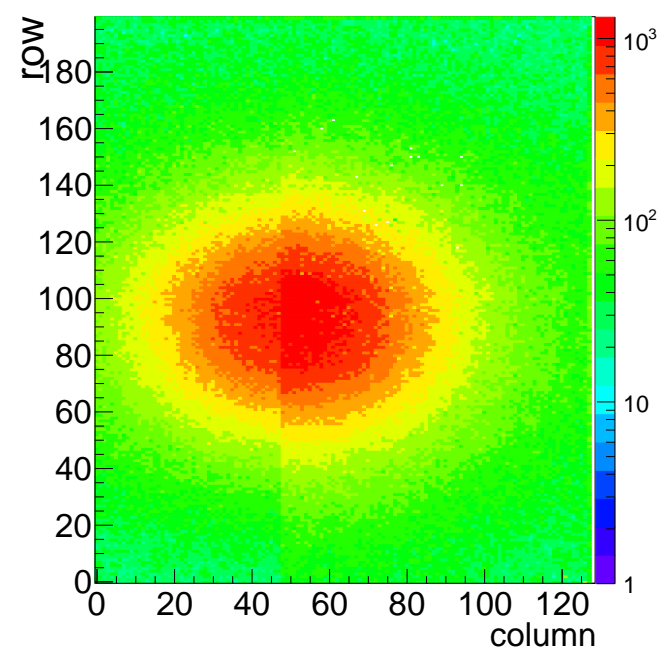

Figure 5: Hitmap of MuPix8 irradiated with a Strontium-90 source. 


\subsection{Module Prototyping}

The ultra thin tracking layers will be produced as a sandwich structure of a polyimide support structure with the sensors glued and sp-TAB bonded to a high density interconnect (HDI) (Fig.6) [10].

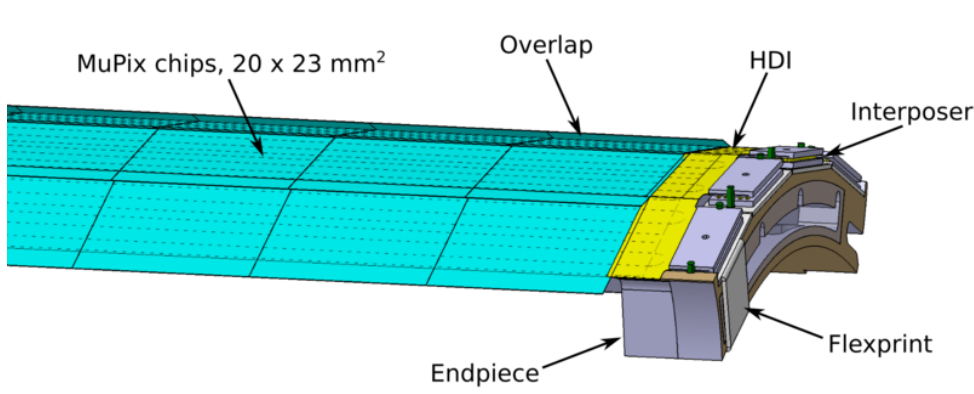

(a) CAD drawing of a pixel detector module.

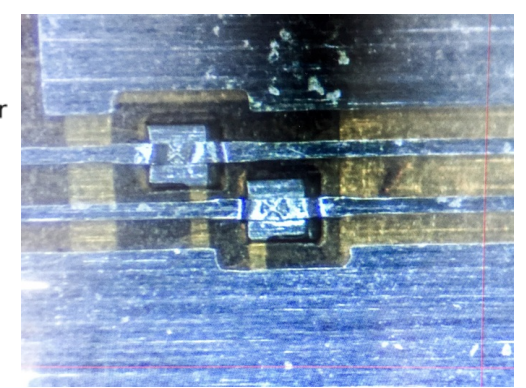

(b) Picture of a Sp-TAB bonding test with a flexprint prototype.

Figure 6: Module prototyping.

Since bond pads will create insensitive area, it is advantageous to place all pads on a single edge of the chip. Insensitive regions can be avoided in the detector design by overlapping sensors.

The MuPix8 chip is the first prototype with all necessary bond pads placed on one edge. That allows to test the chip-internal power distribution and to build first module prototypes with MuPix8 sensors bonded to an HDI.

Additionally, a bandgap reference is implemented as possible voltage reference for the chip. It is designed to stabilize the reference voltage against differences in temperature or supply voltages which will be present in the final detector due to inhomogeneous cooling and voltage drops on the HDI.

\section{Summary \& Outlook}

The architecture of the first large area $1 \times 2 \mathrm{~cm}^{2}$ MuPix 8 prototype, produced in an AMS $180 \mathrm{~nm}$ HV-CMOS process, was presented. It features the full column length and pixel size of the final chip and is readout by an on-chip statemachine, streaming out data on four $1.25 \mathrm{Gbit} / \mathrm{s}$ LVDS data links. Additionally new circuitry is tested which allows for timewalk suppression on-chip or off-chip, aiming for a time resolution better than $10 \mathrm{~ns}$. The prototype is now under characterisation in different labs and on test beam campaigns.

The next prototype MuPix9 is already submitted and will enable tests of novel serial powering circuitry. Furthermore an improved slow control interface is implemented which will enable an easier module integration of the final Mu3e pixel sensor. 


\section{Acknowledgments}

H. Augustin, A. L. Weber and A. Herkert acknowledge support by the HighRR research training group [GRK 2058]. N. Berger thanks the Deutsche Forschungsgemeinschaft for their support and the Mu3e project through an Emmy Noether grant. Further N. Berger thanks the PRISMA Cluster of Excellence for support. S. Dittmeier and L. Huth acknowledge support by the IMPRS-PTFS.

\section{References}

[1] A. Blondel et al., Research Proposal for an Experiment to Search for the Decay $\mu \rightarrow$ eee, ArXiv e-prints (Jan., 2013) , [1301.6113].

[2] I. Perić, A novel monolithic pixelated particle detector implemented in high-voltage CMOS technology, Nucl.Instrum.Meth. A582 (2007) 876.

[3] H. Augustin et al., The MuPix System-on-Chip for the Mu3e Experiment, Nucl. Instrum. Meth. A845 (2017) 194-198, [1603.08751].

[4] H. Augustin, N. Berger, S. Dittmeier, J. Hammerich, U. Hartenstein, Q. Huang et al., MuPix7-A fast monolithic HV-CMOS pixel chip for Muße, Journal of Instrumentation 11 (2016) C11029.

[5] H. Augustin et al., The MuPix Telescope: A Thin, high Rate Tracking Telescope, JINST 12 (2017) C01087, [1611.03102].

[6] H. Augustin et al., Irradiation study of a fully monolithic HV-CMOS pixel sensor design in AMS $180 \mathrm{~nm}, 1712.03921$.

[7] H. Augustin et al., Efficiency and timing performance of the MuPix7 high-voltage monolithic active pixel sensor, Nucl. Instrum. Meth. A902 (2018) 158-163, [1803.01581].

[8] J. Kröger, Readout Hardware for the MuPix8 and a Firmware-based MuPix8 Emulator, master thesis, Heidelberg University, 2017.

[9] A. L. Weber, Entwurf eines Pixelsensorchips für die Teilchenphysik, master thesis, Karlsruher Institut für Technologie, 2016.

[10] N. Berger, S. Dittmeier, L. Henkelmann, A. Herkert, F. M. Aeschbacher, Y. Ng et al., Ultra-low material pixel layers for the muße experiment, Journal of Instrumentation 11 (2016) C12006. 